\title{
Outcome of renal grafts after simultaneous kidney/ pancreas transplantation
}

\author{
G. Hillebrand ${ }^{1}$, W.-D. Illner ${ }^{2}$, D. Abendroth ${ }^{2}$, H. Schneeberger ${ }^{2}$, I. Petry ${ }^{2}$, S. Schleibner ${ }^{2}$, R. Landgraf ${ }^{3}$ and \\ W. Land ${ }^{2}$ \\ ${ }^{1}$ Nephrology Department, Medical Clinic I, Klinikum Grosshadern, ${ }^{2}$ Division of Transplant Surgery, Klinikum Grosshadern and \\ ${ }^{3}$ Medical Clinic "Innenstadt", University of Munich, Munich, FRG
}

Summary. Nineteen patients with endstage renal failure due to Type 1 (insulin-dependent) diabetes mellitus received simultaneous pancreas/kidney transplants using bladder drainage technique. Another group of 25 Type 1 diabetic patients received pancreas/kidney transplants by the duct occlusion technique. We observed a higher incidence of rejection episodes in the patients of the bladder drainage group than those in the duct occlusion group, 14 of 19 patients (74\%) vs 7 of $25(28 \%)$ respectively. Anti CD3 antibodies (Orthoclone ${ }^{\mathrm{R}}$, OKT3) as a part of induction treatment was used more often in the bladder drainage group (58\%) than in the control group (20\%).

Key words: Pancreas/kidney transplantation - Diabetes mellitus - Bladder drainage technique - Renal rejection episode

\section{Introduction}

In recent years, techniques and results in pancreas transplantations have been remarkably improved, although surgical techniques and patient selections are still controversial, especially with respect to long-term results and reversibility of late diabetic complications.

In our first series of simultaneous kidney/pancreas transplantations, relatively poor long-term results of the renal graft were observed when using the duct occlusion technique (Castro 1986, Hillebrand 1987). After modifying the immunosuppressive regimen and the concomitant therapy in the early postoperative phase, the prognosis for the renal graft was improved to the range of kidney transplantation (5-year graft survival rate of $56 \%$ ). Details are described separately in this issue (Illner 1991).
In January 1989 we introduced the bladder drainage technique for pancreas grafting at our centre. In the following we report on our experience with this altered surgical method regarding the kidney graft.

\section{Subjects and methods}

In Group 1 ( $n=25$ patients) simultaneous transplantation was carried out using the duct occlusion technique. The operations were performed between June 1987 and March 1990 prior to changing methods. Group 2 ( $n=19$ patients) also received pancreas/kidney transplants but using the bladder drainage technique. Both groups were comparable with respect to HLA mismatches, preexistent cytotoxic antibodies, age, duration of Type 1 (insulin-dependent) diabetes mellitus and regular dialysis treatment (continuous ambulatory peritoneal dialysis CAPD/hemodialysis). There were slight differences in cold ischaemia time (Group 1:7.5 h, Group 2:13.8 h) as well as in sex distribution (male/female) (Group 1:10/15, Group 2 16/3). All patients were treated with a quadruple drug induction therapy consisting of cyclosporin, azathioprin, steroids and polyclonal sera (antilymphocyte globulin ALG or antithymocyte globulin ATG) or monoclonal serum anti $\mathrm{CD} 3$ antibodies Orthoclone ${ }^{\mathrm{R}}$ OKT3. We evaluated incidence of rejection crises, actuarial 2-year kidney graft survival and patient survival.

\section{Results}

Rejection episodes were observed in 7 of 25 patients $(28 \%)$ in Group 1, and in 14 of 19 patients (74\%), in Group 2 ( $p<0.05$ chi-square test). Steroid resistant rejection crises occurred six times in Group 1 and ten times in Group 2, which necessitated therapy with ALG, ATG or OKT3. Three renal grafts were lost in Group 1, 5 in Group 2. Reasons for graft function loss were in Group 1: 2 patients' death, 1 chronic rejection; in Group 2: 3 acute, 2 chronic rejections.

The 2-year actuarial renal graft survival rate was calculated according to the Kaplan Mayer formula and showed better results in Group $183 \%$ (duct occlusion) than in Group $266 \%$ (bladder drainage). 


\section{Discussion}

In our first series of simultaneous pancreas/kidney transplantation using the bladder drainage technique, 19 patients had a significant higher incidence of renal rejection episodes as well as an unfavourable 2-year renal graft survival in comparison to a historical control group with duct occlusion. This is in contrast to the results of other groups, which are published in this issue. Re-evaluation of the two patient groups showed beside the above-mentioned differences in cold ischaemia time and sex distribution - a higher incidence in post-operative acute renal failure (Group $10 \%$; Group $211 \%$ ), and a frequent use of OKT3 as a part of the induction protocol (Group 1 20\%; Group $258 \%$ ); although differences in the efficacy between ALG vs OKT3 were not described by others (Knechtle 1991).

\section{References}

Castro LA, Landgraf R, Hillebrand G, Land W (1986) Causes of renal graft dysfunction in diabetics after simultaneous pancreas and kidney transplantation. Transpl.Proc. 18:1733-1734

Hillebrand G, Castro LA, Landgraf R, Schleibner S, Illner WD, Abendroth D, Land W (1987) Combined/kidney pancreas transplantation - poor long-term outcome of renal grafts. Transpl.Proc. 19:3909-3910

Illner WD, Abendroth D, Landgraf R, Land W (1991) Long-term results in pancreatic transplantation with special emphasis on the use of prolamin. Diabetologia 34 [Suppl 2]

Knechtle SI, Pirsch JD, Groshek M, et al. (1991) OKT3 vs ALG induction therapy in combined pancreas-kidney transplantation. Transpl.Proc. 23:1581-1582

Dr. G. Hillebrand Nephrology Department, Med. Klinik I, Klinikum Grosshadern, University of Munich

P.O. Box 701260

8000 Munich 70, F.R.G. 